\title{
Backface Culling for Motion Blur and Depth of Field
}

\author{
Jacob Munkberg and Tomas Akenine-Möller \\ Intel Corporation and Lund University \\ October 2010
}

\begin{abstract}
For triangles with linear vertex motion, common practice is to backface cull a triangle if it is backfacing at both the start and end of the motion. However, this is not conservative. We derive conservative tests that guarantee that a moving triangle is backfacing over an entire time interval and over the area of a lens. In addition, we present tests for the special cases of only motion blur and only depth of field. Our techniques apply to real-time and offline rendering, and to both stochastic point sampling and analytical visibility methods. The rendering errors introduced by the previous technique can easily be detected for large defocus blur, but in the majority of cases, the errors introduced are hard to detect. We conclude that our tests are needed if one needs guaranteed artifact-free images. Finally, as a side result, we derive time-continuous Bézier edge equations.
\end{abstract}

\section{Introduction}

One of the most important culling tests is backface culling. When rendering motion blur and depth of field, an excessive amount of inside tests can be generated, and therefore, accurate backface culling tests are needed also for these contexts. For moving triangles, where each vertex moves along a line in three dimensions, a commonly used technique assumes that a moving triangle is backfacing over the entire time interval if the triangle is backfacing at the start and end of the motion. However, this is not true in the general case, and certainly, this has been known in some groups, but we are not aware of any documentation of this, nor of any previous solution. We derive tests for the special cases of using either only motion blur or only depth of field. The combination of these effects is harder to handle, and therefore, we derive a coarser conservative test for motion blur with depth of field.

Let us start with a motivating example that clearly shows that a triangle can be backfacing at the start of the motion (at $t=0$ ), then turns frontfacing, and then again is backfacing at the end of the motion (at $t=1)$. Consider a triangle with vertices in $x y w$ space as follows:

$$
\mathbf{p}_{0}=(\alpha-t, 0,1), \quad \mathbf{p}_{1}=(0, t-\beta, 1), \quad \mathbf{p}_{2}=(0, \beta-t, 1),
$$

where $\alpha, \beta \in[0,1], \alpha<\beta$ are constants, and $t$ is the time parameter. Each triangle vertex moves linearly in time, and the triangle is shown at three different times Figure 1. In the same figure, the swept surface is also illustrated. The sign of the determinant of the three points determines whether the triangle can be backface culled [McCool et al. 02]:

$$
\mathbf{p}_{0} \cdot\left(\mathbf{p}_{1} \times \mathbf{p}_{2}\right)=-2(\alpha-t)(\beta-t),
$$




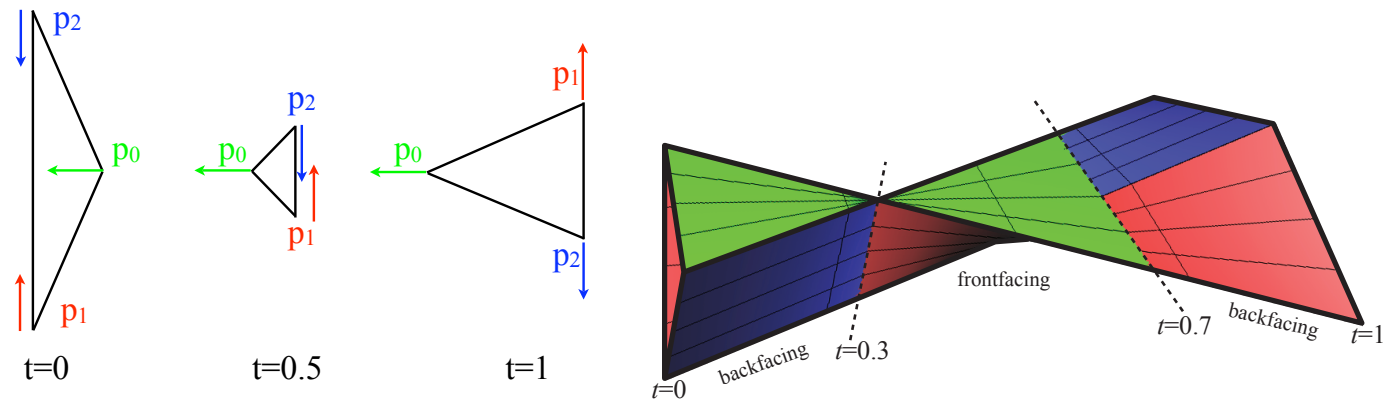

Figure 1: Left: The triangle is backfacing at $t=0$ and $t=1$, and frontfacing at $t=0.5$. Right: The surfaces that the moving triangle sweeps out are visualized, and one can see that the "edge surfaces" intersect when the facing changes. This happens at $t=\alpha=0.3$ and $t=\beta=0.7$.

where a negative sign indicates a backfacing triangle. Given $\alpha<\beta$, we note that the triangle is frontfacing when $t \in[\alpha, \beta]$, even though the triangle is backfacing at $t=0$ and $t=1$. At $t=\alpha$ and $t=\beta$, the triangle is degenerate, i.e., it is here that the switch from backfacing to frontfacing or vice versa occurs. A stochastically rasterized example of this configuration is shown to the left in Figure 2.

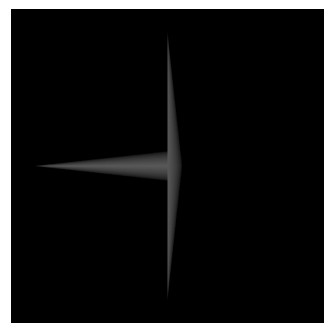

backfacing

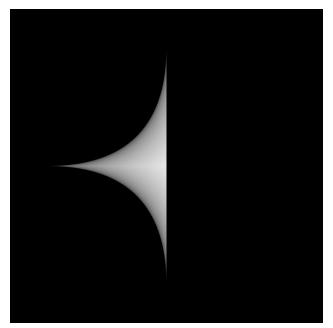

frontfacing

A: Quadratic BF function

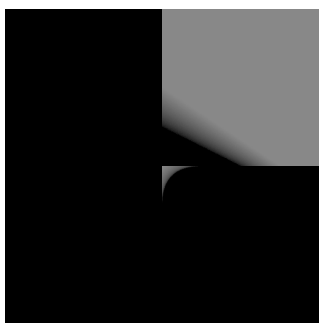

backfacing

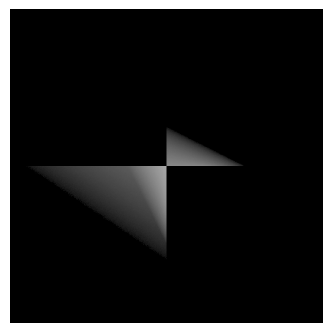

frontfacing

B: Cubic BF function

Figure 2: Motion blur rasterized triangles. The shutter is opened in $t \in[0,1]$. A: The triangle moving according to Equation 1 is rasterized on screen with $\alpha=0.1$ and $\beta=0.9$, where the triangle is backfacing at $t=0$ and $t=1$. The left image shows the covered samples for back faces only, while the right image shows covered samples for front faces. Note that with naïve backface culling, all the covered samples to the right will be missed, which is incorrect. B: The triangle moving according to Equation 3 flips from front to backfacing (or vice versa) three times in the time interval. The left image shows a rendering of the backfacing region, and the right image shows the frontfacing region.

In general, the determinant is a cubic polynomial, which can be seen when considering the following time-continuous triangle:

$$
\mathbf{p}_{0}=(\alpha-t, 0,1), \quad \mathbf{p}_{1}=(0, \beta-t, 1), \quad \mathbf{p}_{2}=(0,0, \gamma-t)
$$


where $\alpha, \beta, \gamma \in[0,1]$ are constants, and $t$ is the time parameter. The determinant then becomes:

$$
\mathbf{p}_{0} \cdot\left(\mathbf{p}_{1} \times \mathbf{p}_{2}\right)=(\alpha-t)(\beta-t)(\gamma-t),
$$

which has three real roots in the interval $t \in[0,1]$. This means that the facing can change three times. An illustration of such a triangle is shown to the right in Figure 2. At this point, we have motivated the need for a more correct test, and derivations for such tests follow in the subsequent sections.

\section{Backface Culling for Motion Blur}

In general, we follow the notation from previous work in the field of stochastic [Akenine-Möller et al. 07] and analytical rasterization [Gribel et al. 10]. Assume that we have a moving triangle, where the vertices move linearly within a frame, from time $t=0$ to $t=1$. At $t=0$, we denote the the vertices $\mathbf{q}_{i}$ and at $t=1$ we call them $\mathbf{r}_{i}$. We work in clip space, using $2 \mathrm{D}$ homogeneous coordinates $(2 \mathrm{DH})$, so a vertex is defined as $\mathbf{p}=\left(p_{x}, p_{y}, p_{w}\right)$ [Olano and Greer 97]. A linearly interpolated vertex is then expressed as:

$$
\mathbf{p}_{i}(t)=(1-t) \mathbf{q}_{i}+t \mathbf{r}_{i} .
$$

Given a moving triangle with vertices $\left(\mathbf{p}_{0}(t), \mathbf{p}_{1}(t), \mathbf{p}_{2}(t)\right)$, we form the matrix:

$$
\mathbf{M}(t)=\left[\begin{array}{lll}
p_{0_{x}} & p_{1_{x}} & p_{2_{x}} \\
p_{0_{y}} & p_{1_{y}} & p_{2_{y}} \\
p_{0_{w}} & p_{1_{w}} & p_{2_{w}}
\end{array}\right],
$$

where we have omitted the temporal dependence for readability. The triangle can be backface culled if $\operatorname{det}(\mathbf{M})<0$, where the determinant is expressed as [McCool et al. 02]:

$$
\operatorname{det}(\mathbf{M})=\mathbf{p}_{0} \cdot\left(\mathbf{p}_{1} \times \mathbf{p}_{2}\right) .
$$

Geometrically, this can be interpreted as a (scaled) signed volume computation of the tetrahedron spanned by the origin and the triangle. Thus, we want to determine if $\mathbf{p}_{0}(t) \cdot\left(\mathbf{p}_{1}(t) \times\right.$ $\left.\mathbf{p}_{2}(t)\right)<0$ for $t \in[0,1]$. The cross product of two linearly moving vertices can be expanded as [Akenine-Möller et al. 07]:

$$
\mathbf{p}_{1} \times \mathbf{p}_{2}=\left((1-t) \mathbf{q}_{1}+t \mathbf{r}_{1}\right) \times\left((1-t) \mathbf{q}_{2}+t \mathbf{r}_{2}\right)=t^{2} \mathbf{f}+t \mathbf{g}+\mathbf{h},
$$

where:

$$
\begin{aligned}
\mathbf{f} & =\left(\mathbf{r}_{1}-\mathbf{q}_{1}\right) \times\left(\mathbf{r}_{2}-\mathbf{q}_{2}\right), \\
\mathbf{g} & =\left(\mathbf{r}_{1}-\mathbf{q}_{1}\right) \times \mathbf{q}_{2}-\left(\mathbf{r}_{2}-\mathbf{q}_{2}\right) \times \mathbf{q}_{1}, \\
\mathbf{h} & =\mathbf{q}_{1} \times \mathbf{q}_{2} .
\end{aligned}
$$

Using this expression, we can derive the time-dependent determinant:

$$
\begin{aligned}
\operatorname{det}(\mathbf{M}) & =\mathbf{p}_{0}(t) \cdot\left(\mathbf{p}_{1}(t) \times \mathbf{p}_{2}(t)\right) \\
& =\left((1-t) \mathbf{q}_{0}+t \mathbf{r}_{0}\right) \cdot\left(t^{2} \mathbf{f}+t \mathbf{g}+\mathbf{h}\right) \\
& =a t^{3}+b t^{2}+c t+d,
\end{aligned}
$$


where:

$$
\begin{aligned}
a & =\left(\mathbf{r}_{0}-\mathbf{q}_{0}\right) \cdot \mathbf{f}, \\
b & =\left(\mathbf{r}_{0}-\mathbf{q}_{0}\right) \cdot \mathbf{g}+\mathbf{q}_{0} \cdot \mathbf{f}, \\
c & =\left(\mathbf{r}_{0}-\mathbf{q}_{0}\right) \cdot \mathbf{h}+\mathbf{q}_{0} \cdot \mathbf{g}, \\
d & =\mathbf{q}_{0} \cdot \mathbf{h} .
\end{aligned}
$$

Note that the coefficient $d=\mathbf{q}_{0} \cdot \mathbf{h}=\mathbf{q}_{0} \cdot\left(\mathbf{q}_{1} \times \mathbf{q}_{2}\right)$ is the backface test for the triangle at $t=0$. Also, the value of the polynomial at $t=1$ is $a+b+c+d=\mathbf{r}_{0} \cdot\left(\mathbf{r}_{1} \times \mathbf{r}_{2}\right)$, which, analogously, is the backface test at $t=1$. The expression for the coefficient:

$$
a=\left(\mathbf{r}_{0}-\mathbf{q}_{0}\right) \cdot\left[\left(\mathbf{r}_{1}-\mathbf{q}_{1}\right) \times\left(\mathbf{r}_{2}-\mathbf{q}_{2}\right)\right],
$$

is the determinant test for the motion vectors of the three vertices, and if they all lie in the same plane, the cubic term is zero, i.e., $a=0$. Thus, it is only when the motion vectors span a volume in $2 \mathrm{DH}$ that the determinant will be a cubic function.

We note that if the polynomial does not have any roots in $t \in[0,1]$ and $d<0$, then the triangle can be safely backface culled.

Optimization for Motion Along a Vector If the triangle's motion vectors are parallel, such that:

$$
\mathbf{p}_{i}(t)=(1-t) \mathbf{q}_{i}+t \mathbf{r}_{i}=\mathbf{q}_{i}+t \gamma_{i} \mathbf{d},
$$

where $\gamma_{i} \in \mathbb{R}$, the computations can be simplified. It follows that $\mathbf{f}=\left(\mathbf{q}_{1}-\mathbf{r}_{1}\right) \times\left(\mathbf{q}_{2}-\mathbf{r}_{2}\right)=$ $\gamma_{1} \gamma_{2} \mathbf{d} \times \mathbf{d}=0$, and the cubic coefficient $a=0$. Using $\mathbf{f}=0$ and Equation 13, we can show that the coefficient $b$ is zero when the motion vectors are parallel:

$$
\begin{aligned}
b & =\left(\mathbf{r}_{0}-\mathbf{q}_{0}\right) \cdot \mathbf{g}+\mathbf{q}_{0} \cdot \mathbf{f} \\
& \left.\left.=\left(\mathbf{r}_{0}-\mathbf{q}_{0}\right) \cdot\left(\left(\mathbf{r}_{1}-\mathbf{q}_{1}\right) \times \mathbf{q}_{2}\right)-\left(\mathbf{r}_{2}-\mathbf{q}_{2}\right) \times \mathbf{q}_{1}\right)\right) \\
& =\gamma_{0} \gamma_{1} \mathbf{d} \cdot\left(\mathbf{d} \times\left(\mathbf{q}_{2}-\mathbf{q}_{1}\right)\right)=0 .
\end{aligned}
$$

Thus, for motion along a common direction, including pure translation, the determinant is a linear function in $t$, and given that the value of the determinant at both $t=0$ and $t=1$ is negative, the triangle can be backface culled.

\subsection{Practical Backface Culling Test}

The cubic coefficient $a$ (Equation 12) of the backface function is the determinant of the triangle's three motion vectors, which are often small or near parallel. Therefore, directly computing the backfacing function on power form (Equation 10) can be numerically unstable. To alleviate the problem, we note that a vertex moving linearly in time as in Equation 5 is a Bézier curve of degree 1. Furthermore, the time-dependent edge equations are cross products of linearly moving vertices, and can also be expressed on Bernstein form as:

$$
\mathbf{p}_{i}(t) \times \mathbf{p}_{j}(t)=(1-t)^{2} \mathbf{c}_{0}+2(1-t) t \mathbf{c}_{1}+t^{2} \mathbf{c}_{2},
$$

where:

$$
\mathbf{c}_{0}=\mathbf{q}_{i} \times \mathbf{q}_{j}, \quad \mathbf{c}_{1}=\frac{1}{2}\left(\mathbf{q}_{i} \times \mathbf{r}_{j}+\mathbf{r}_{i} \times \mathbf{q}_{j}\right) \quad \text { and } \quad \mathbf{c}_{2}=\mathbf{r}_{i} \times \mathbf{r}_{j}
$$


Hence, the full edge equation is expressed on Bernstein form as shown below:

$$
e(t, x, y)=\left((1-t)^{2} \mathbf{c}_{0}+2(1-t) t \mathbf{c}_{1}+t^{2} \mathbf{c}_{2}\right) \cdot(x, y, 1)
$$

The backfacing function, given by the determinant (Equation 10) expressed on cubic Bernstein form then becomes:

$$
\operatorname{det}(\mathbf{M}(t))=\sum_{i=0}^{3} b_{i}\left(\begin{array}{l}
3 \\
i
\end{array}\right)(1-t)^{3-i} t^{i}
$$

with coefficients, $b_{i}$, given by:

$$
\begin{aligned}
b_{0} & =\mathbf{q}_{0} \cdot\left(\mathbf{q}_{1} \times \mathbf{q}_{2}\right) \\
b_{1} & =1 / 3\left[\mathbf{q}_{0} \cdot\left(\mathbf{q}_{1} \times \mathbf{r}_{2}+\mathbf{r}_{1} \times \mathbf{q}_{2}\right)+\mathbf{r}_{0} \cdot\left(\mathbf{q}_{1} \times \mathbf{q}_{2}\right)\right] \\
b_{2} & =1 / 3\left[\mathbf{r}_{0} \cdot\left(\mathbf{q}_{1} \times \mathbf{r}_{2}+\mathbf{r}_{1} \times \mathbf{q}_{2}\right)+\mathbf{q}_{0} \cdot\left(\mathbf{r}_{1} \times \mathbf{r}_{2}\right)\right] \\
b_{3} & =\mathbf{r}_{0} \cdot\left(\mathbf{r}_{1} \times \mathbf{r}_{2}\right)
\end{aligned}
$$

Note that there are no vector subtractions involved in these expressions, which avoids the precision issue of directly computing $a$ in Equation 10. We exploit the convex hull property of the Bernstein basis for our test. By simply checking if all of the coefficients, $b_{i}, i \in\{0,1,2,3\}$, are negative, we know that the triangle is conservatively backfacing. This is a coarser test than testing against the true maximum of the cubic polynomial, but reduces the risk of numerical precision issues. Note that $b_{0}$ and $b_{3}$ are the backface tests at $\mathrm{t}=0$ and $\mathrm{t}=1$ respectively. The test can be refined by applying de Casteljau steps to the coefficients and testing if any generated coefficient is positive.

\subsection{Higher-Order Vertex Motion}

If the triangle vertex motion can be expressed as a polynomial, we can generalize the previous test. We express the motion of each triangle vertex as a Bézier curve of degree $n$ in 2DH:

$$
\mathbf{p}_{i}(t)=\sum_{j=0}^{n} \mathbf{b}_{j}^{i} B_{j}^{n}(t) .
$$

The backface test then becomes:

$$
\begin{aligned}
\operatorname{det}(\mathbf{M}(t)) & =\mathbf{p}_{0}(t) \cdot\left(\mathbf{p}_{1}(t) \times \mathbf{p}_{2}(t)\right) \\
& =\sum_{i=0}^{n} \mathbf{b}_{i}^{0} B_{i}^{n}(t) \cdot\left(\sum_{j=0}^{n} \mathbf{b}_{j}^{1} B_{j}^{n}(t) \times \sum_{k=0}^{n} \mathbf{b}_{k}^{2} B_{k}^{n}(t)\right) \\
& =\sum_{i, j, k=0}^{n} B_{i+j+k}^{3 n} \frac{\left(\begin{array}{c}
n \\
i
\end{array}\right)\left(\begin{array}{c}
n \\
j
\end{array}\right)\left(\begin{array}{l}
n \\
k
\end{array}\right)}{\left(\begin{array}{c}
3 n \\
i+j+k
\end{array}\right)} \mathbf{b}_{i}^{0} \cdot\left(\mathbf{b}_{j}^{1} \times \mathbf{b}_{k}^{2}\right)
\end{aligned}
$$

This is a Bézier curve of degree $3 n$, where the control points are sums of scaled determinants of three control points, one from each of the three curves describing the vertex motion. A conservative backface test can again be derived by using the convex hull property and testing the sign of the control points. As expected, we obtain Equation 18 for the linear motion case, i.e., when $n=1$. 


\subsection{Backface Culling for Motion Blur in Screen Space}

For screen-space rasterization, a common backface test is given by the sign of the screen-space area of the triangle. Let us define two edges of the projected triangle as:

$$
\mathbf{e}_{1}(t)=\frac{\mathbf{p}_{1}(t)}{p_{1_{w}}(t)}-\frac{\mathbf{p}_{0}(t)}{p_{0_{w}}(t)}, \quad \mathbf{e}_{2}(t)=\frac{\mathbf{p}_{2}(t)}{p_{2_{w}}(t)}-\frac{\mathbf{p}_{0}(t)}{p_{0_{w}}(t)} .
$$

Twice the signed area can now be expressed as:

$$
2 A(t)=\mathbf{e}_{1}(t) \times \mathbf{e}_{2}(t)=\frac{p_{2_{w}} \mathbf{p}_{0} \times \mathbf{p}_{1}+p_{0_{w}} \mathbf{p}_{1} \times \mathbf{p}_{2}+p_{1_{w}} \mathbf{p}_{2} \times \mathbf{p}_{0}}{p_{0_{w}} p_{1_{w}} p_{2_{w}}} .
$$

Recall that that each vertex is a function of $t$ (Equation 5), which results in that the backface test in screen space is a cubic rational function in $t$. The triangle moves in a plane, but the vertex positions are no longer linearly interpolated in $t$ and the triangle can change facing at most three times.

The magnitude of the denominator $p_{0_{w}} p_{1_{w}} p_{2_{w}}$ is irrelevant for the area test, so if we know the signs of the vertices $w$ components after clipping, the denominator can be omitted. Similar to the homogeneous case, the signed area is a cubic polynomial.

Linear motion in screen space For this case, the area function becomes: $2 A(t)=\left(\overline{\mathbf{p}}_{1}(t)-\right.$ $\left.\overline{\mathbf{p}}_{0}(t)\right) \times\left(\overline{\mathbf{p}}_{2}(t)-\overline{\mathbf{p}}_{0}(t)\right)$, where $\overline{\mathbf{p}}_{i}$ are moving vertices derived from linearly interpolating the projected start $\left(\overline{\mathbf{q}}_{i}\right)$ and end $\left(\overline{\mathbf{r}}_{i}\right)$ vertices. This is a quadratic polynomial, so even in this case, the moving triangle can be backfacing at $t=0$ and $t=1$, and still be frontfacing somewhere in between. This is precisely the scenario illustrated in Figure 1.

\section{Backface Culling for Depth of Field}

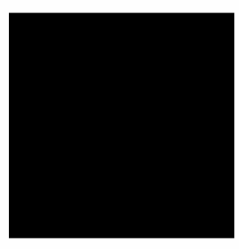

$\mathrm{F}=$ near

BF cull at center of lens

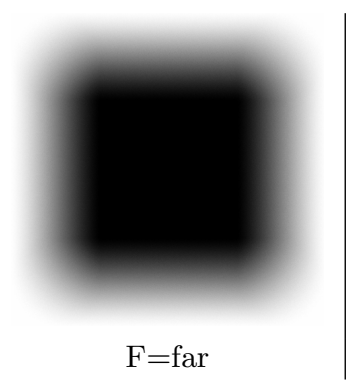

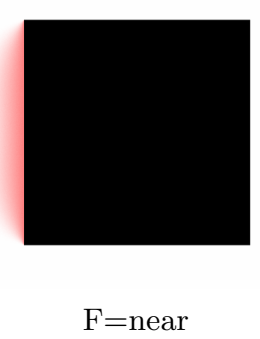

Conservative BF cull

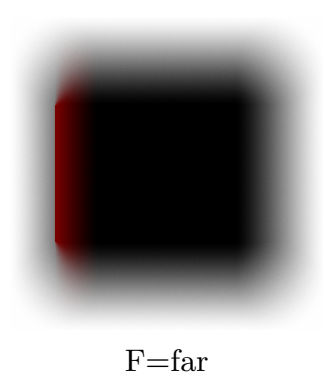

$$
\text { ull }
$$

Figure 3: A red cube with a black front face is rendered with depth of field. Focus is set at the front and back of the cube. The cube is positioned so that the left face has a normal perpendicular to the view vector at the center of the lens. The left face can thus be backface culled for that camera position. For rays starting slightly off-center, the left (red) side is visible, and contributes to the final image. The two leftmost images show the result when the backface test is done only at the center of the lens, and the two rightmost images the reference result. 
A naïve backface test for depth of field is to only check the backface status at the center of the lens. Figure 3 shows that this is not correct. Depth of field is a shear in clip space [Cook et al. 84]. This shear can be represented by applying the matrix [Ragan-Kelley et al. ]:

$$
\mathbf{S}(u, v)=\left(\begin{array}{cccc}
1 & 0 & -H u / J & H u \\
0 & 1 & -I v / J & I v \\
0 & 0 & 1 & 0 \\
0 & 0 & 0 & 1
\end{array}\right),
$$

to the clip space coordinates of the triangle. $H, I$ and $J$ are constants given by the location of the focal plane, the camera aperture size and the near \& far plane. The location on the lens is given by $(u, v)$. Applying this matrix to a three-dimensional homogeneous vertex, $\hat{\mathbf{p}}_{i}=\left(p_{i_{x}}, p_{i_{y}}, p_{i_{z}}, p_{i_{w}}\right)$, in clip space results in a sheared position

$$
\hat{\mathbf{s}}_{i}(u, v)=\left(p_{i_{x}}+\alpha_{i} u, p_{i_{y}}+\beta_{i} v, p_{i_{z}}, p_{i_{w}}\right)
$$

where $\alpha_{i}=-H / J p_{i_{z}}+H p_{i_{w}}$ and $\beta_{i}=-I / J p_{i_{z}}+I p_{i_{w}}$. To simplify notation below, we introduce $\mathbf{l}_{i}=\left(\alpha_{i} u, \beta_{i} v, 0\right)$ and let $\mathbf{s}_{i}(u, v)$ and $\mathbf{p}_{i}(u, v)$ denote the two-dimensional homogeneous vertices, consisting of the $x y w$ components of $\hat{\mathbf{s}}_{i}(u, v)$ and $\hat{\mathbf{p}}_{i}$ respectively, that is:

$$
\mathbf{s}_{i}(u, v)=\mathbf{p}_{i}+\mathbf{l}_{i}(u, v) .
$$

Note that $\mathbf{l}_{i} \times \mathbf{l}_{j}=u v\left(0,0, \alpha_{i} \beta_{j}-\alpha_{j} \beta_{i}\right)=0$ since $\alpha_{i}=\frac{H}{I} \beta_{i}$. The backfacing criterion then becomes:

$$
\begin{aligned}
\operatorname{det}(\mathbf{M}(u, v))= & \mathbf{s}_{0} \cdot\left(\mathbf{s}_{1} \times \mathbf{s}_{2}\right) \\
= & \mathbf{p}_{0} \cdot\left(\mathbf{p}_{1} \times \mathbf{p}_{2}\right)+\mathbf{l}_{0} \cdot\left(\mathbf{p}_{1} \times \mathbf{p}_{2}\right) \\
& +\mathbf{l}_{1} \cdot\left(\mathbf{p}_{2} \times \mathbf{p}_{0}\right)+\mathbf{l}_{2} \cdot\left(\mathbf{p}_{0} \times \mathbf{p}_{1}\right) \\
= & a u+b v+c .
\end{aligned}
$$

The coefficients are given by:

$$
\begin{aligned}
a & =\alpha_{0}\left(\mathbf{p}_{1} \times \mathbf{p}_{2}\right)_{x}+\alpha_{1}\left(\mathbf{p}_{2} \times \mathbf{p}_{0}\right)_{x}+\alpha_{2}\left(\mathbf{p}_{0} \times \mathbf{p}_{1}\right)_{x} \\
b & =\beta_{0}\left(\mathbf{p}_{1} \times \mathbf{p}_{2}\right)_{y}+\beta_{1}\left(\mathbf{p}_{2} \times \mathbf{p}_{0}\right)_{y}+\beta_{2}\left(\mathbf{p}_{0} \times \mathbf{p}_{1}\right)_{y} \\
c & =\mathbf{p}_{0} \cdot\left(\mathbf{p}_{1} \times \mathbf{p}_{2}\right)
\end{aligned}
$$

The triangle will change its facing somewhere on a circular lens with radius $R$ only if there is a solution to the following system of equations:

$$
\begin{aligned}
a u+b v+c & =0, \\
u^{2}+v^{2} & <R^{2},
\end{aligned}
$$

Geometrically, this is an intersection between a circle and a line, which has solutions only if $c^{2}<$ $R^{2}\left(a^{2}+b^{2}\right)$. Consequently, if the triangle is backfacing at the center of the lens $(c<0)$ and there are no face changes when moving over the lens, the triangle can be backface culled. More formally, if:

$$
\begin{aligned}
c & <0, \quad \text { and } \\
c^{2} & >R^{2}\left(a^{2}+b^{2}\right),
\end{aligned}
$$

the triangle can be conservatively backface culled. Intuitively, the triangle changes facing over the lens only if the triangle's plane equation (in three dimensions) intersects the shape of the lens. 


\section{Conservative Backface Culling in 5D}

By multiplying the moving vertex in Equation 5 with the shear matrix, $\mathbf{S}$, in Equation 22, the resulting vertex displacement from motion and depth of field, $\mathbf{o}(u, v, t)$, is obtained:

$$
\begin{aligned}
& \hat{\mathbf{o}}_{i}(u, v, t)=\mathbf{S}(u, v) \hat{\mathbf{p}}_{i}(t)=\mathbf{S}(u, v)\left((1-t) \hat{\mathbf{q}}_{i}+t \hat{\mathbf{r}}_{i}\right), \\
& \mathbf{o}_{i}(u, v, t)=\left(p_{i_{x}}(t)+\alpha_{i}(t) u, p_{i_{y}}(t)+\beta_{i}(t) v, p_{i_{w}}(t)\right),
\end{aligned}
$$

where $\alpha_{i}(t)=H / J p_{i_{z}}(t)+H p_{i w}(t)$ and $\beta_{i}(t)=I / J p_{i_{z}}(t)+I p_{i_{w}}(t)$ are linear functions in $t$.

The corresponding backface test from Equation 25 is now expressed as:

$$
\operatorname{det}(\mathbf{M}(u, v, t))=\mathbf{o}_{0} \cdot\left(\mathbf{o}_{1} \times \mathbf{o}_{2}\right)=a(t) u+b(t) v+c(t) .
$$

The coefficients $a(t), b(t)$, and $c(t)$ are cubic functions in $t$, and following Equation 28, the triangle can be conservatively backface culled when:

$$
\begin{aligned}
c(t) & <0, \quad \text { and } \\
c^{2}(t) & >R^{2}\left(a^{2}(t)+b^{2}(t)\right), \quad t \in[0,1] .
\end{aligned}
$$

\subsection{Practical 5D Backface Culling Test}

In this section, we sketch a practical implementation of the backface culling test for motion blurred and defocused triangles.

Solving Equation 31 using a numerical root solver is unlikely to be worth the effort. Instead, interval arithmetic [Moore 66] can be used, where we denote an interval $\hat{c}=\left[\min _{t}(c), \max _{t}(c)\right]=$ $[\underline{c}, \bar{c}]$. A conservative test is then:

$$
\begin{aligned}
\bar{c} & <0, \quad \text { and } \\
\bar{c}^{2} & >R^{2}\left(\max \left(a^{2}+b^{2}\right)\right), \quad t \in[0,1]
\end{aligned}
$$

where we used $\bar{c}<0 \Rightarrow \min \left(\underline{c}^{2}, \bar{c}^{2}\right)=\bar{c}^{2}$ to simplify the second condition. We first note that a coarse, but fast approximation of the upper bound of $a^{2}(t)+b^{2}(t)$ is given by:

$$
\max _{t \in[0,1]}\left(a^{2}+b^{2}\right) \leq \max _{t \in[0,1]}\left(\underline{a}^{2}, \bar{a}^{2}\right)+\max _{t \in[0,1]}\left(\underline{b}^{2}, \bar{b}^{2}\right),
$$

which essentially is a Manhattan distance approximation.

In the scenes tested, the $t^{3}$ and $t^{2}$ terms of the polynomials $a(t)$ and $b(t)$ are close to zero for most triangles. This implies that the $a(t), b(t)$ and $c(t)$ terms in Equation 32 are approximately linear, but also that care must be taken to avoid precision issues. By using first-order Taylor models [Makino and Berz 03], we ensure stability when the $t^{3}$ and $t^{2}$ terms are small, but preserves the linear dependence in $t$. An arbitrary cubic polynomial is bounded over $t \in[0,1]$ by:

$$
k_{3} t^{3}+k_{2} t^{2}+k_{1} t+k_{0} \approx k_{1} t+k_{0}+\hat{r}_{k},
$$

where $\hat{r}$ is a remainder interval which bounds the quadratic and cubic terms:

$$
\hat{r}_{k}=\left[-\left|k_{3}\right|-\left|k_{2}\right|,\left|k_{3}\right|+\left|k_{2}\right|\right] \text {. }
$$


We use this to conservatively express $a^{2}(t)+b^{2}(t)$ as:

$$
a^{2}(t)+b^{2}(t) \approx\left(a_{1} t+a_{0}+\hat{r}_{a}\right)^{2}+\left(b_{1} t+b_{0}+\hat{r}_{b}\right)^{2} .
$$

A conservative upper bound is given by:

$$
\max _{t \in[0,1]}\left(a^{2}(t)+b^{2}(t)\right) \leq a_{1}^{2}+b_{1}^{2}+2 \max \left(0, a_{0} a_{1}+b_{0} b_{1}\right)+a_{0}^{2}+b_{0}^{2}+\bar{r}_{a^{2}+b^{2}}
$$

where $\bar{r}_{a^{2}+b^{2}}$ is a linear function in the remainder intervals $\hat{r}_{a}$ and $\hat{r}_{b}$. If $a(t)$ and $b(t)$ are linear in $t, \hat{r}_{a}=\hat{r}_{b}=0$ and $\bar{r}_{a^{2}+b^{2}}=0$. We use the backface test for motion blur to determine the backface status at the center of the lens as described above. The final test is given by the conditions in Equation 32, where $a^{2}(t)+b^{2}(t)$ is bounded using Equation 37 .

Square Lens Approximation If we approximate the lens with a square with side length $2 R$, we get a coarser backfacing conditions given by the equations:

$$
\begin{aligned}
\bar{c} & <0, \quad \text { and } \\
\max ( \pm R(a \pm b)+c) & <0, \quad t \in[0,1] .
\end{aligned}
$$

However, our experiments show that Equation 32 using the Taylor model bounds (Equation 37) has slightly higher cull rates, as the vast majority of false backfacing decisions are due to DOF. This implies that the test should be optimized for DOF.

\section{$5 \quad$ Results}

In this section, statistics are gathered from a number of key-framed animations taken from the Utah Animation Repository ${ }^{1}$ and the UNC Dynamic Scene Benchmark. ${ }^{2}$ We denote the test of only checking backface status at the start and end time as STARTEND in the evaluations below.

\subsection{Cost Estimation}

The cost in scalar instructions of the different tests are given in Table 1, with the assumption that the edge equation coefficients (Equation 15) can be reused. For depth of field, the coefficients $\alpha_{i}$ and $\beta_{i}$ are triangle constants and are not included in the cost. The correct backface test costs less than a single inside tests for the motion blur only or DOF only case. For the full 5D test, we do not use 5D edge equations, but instead position the triangle for each uvt tuple. Therefore, no triangle setup computations can be reused, and the backface test becomes considerably more expensive. As a lower limit, a $5 \mathrm{D}$ backface test with coarse bounds of the cubic polynomials involved in the expressions, costs $>3 \times$ of a single $5 \mathrm{D}$ inside test.

\subsection{Motion Blur}

All examples were rendered in $512 \times 512$ pixels using 64 samples per pixel. The motion blur test scenes are shown in Figure 4 with three different motion blur settings. For each animation, we

\footnotetext{
${ }^{1}$ http://www.sci.utah.edu/ wald/animrep/

${ }^{2}$ http://gamma.cs.unc.edu/DYNAMICB/
} 


\begin{tabular}{l|c|c|c|c||c|c} 
Instr. & INSIDE & STARTEND & BFMB & BFDOF & INSIDE5D & BF5D \\
\hline MAD/MSUB & 24 & 4 & 12 & 7 & 39 & 100 \\
MUL & - & 2 & 6 & 6 & 27 & 54 \\
ADD/SUB & - & - & 3 & - & 1 & 60 \\
CMP & 3 & 2 & 4 & 1 & 3 & 2 \\
MIN/ABS & - & - & - & - & - & 14 \\
\hline SUM: & $\mathbf{2 7}$ & $\mathbf{8}$ & $\mathbf{2 5}$ & $\mathbf{1 4}$ & $\mathbf{7 0}$ & $>\mathbf{2 3 0}$
\end{tabular}

Table 1: Cost of correct backface tests for motion blur and depth of field. Inside reports the cost for one per-sample inside test (without tiebreaker rules). InSIDE5D is the positioning and inside test for full 5D rasterization. BF5D denotes the 5D backface test with depth of field and motion blur, and is a lower estimate with coarse bounds of the involved cubic functions.

report the percentage of triangles potentially visible, but rejected by the STARTEND test. The cubic backfacing function is bounded using a subdivided Bézier control cage (using 10 control points), so the percentage reported is slightly higher than the actual miss rate. The number of affected fragments are reported by the per-sample inside test and is an exact measure of the impact on the final result. The results are presented in Table 2.

The Ben animation shows a running man with 78029 triangles. Cloth contains complex motion and a large triangle count (92230). Wood Doll is a simple rigid body animation with modest triangle count (5378 triangles). A camera motion has been added to highlight the common case when both the object and the camera are animated. Figure 5 shows a zoomed in version of the animation with camera rotation. Using a perceptual metric [Yee 04], $2 \%$ of the pixels differ for this particular frame.

For motion blur, the number of triangles falsely rejected by the STARTEnd test is modest, and the visual impact is negligible. It is indeed possible to construct cases where this matters (see Section 1), but for many practical examples with small triangles, closed meshes and limited motion per frame, we conclude that the STARTEND backface test may be sufficient.

\begin{tabular}{l||c|c||c|c||c|c}
\multicolumn{1}{c||}{} & \multicolumn{2}{c||}{$\Delta=1$} & \multicolumn{2}{c||}{$\Delta=5$} & \multicolumn{2}{c}{$\Delta=10$} \\
Name & rej.tris (\%) & $\bar{s}$ & rej.tris $(\%)$ & $\bar{s}$ & rej.tris (\%) & $\bar{s}$ \\
\hline Ben & 0.1 & 13 & 0.2 & 726 & 0.7 & 4450 \\
Cloth & 0.004 & 78 & 0.02 & 425 & 0.1 & 4162 \\
Wood Doll & 0 & 0 & 0.1 & 532 & 0.3 & 1539
\end{tabular}

Table 2: Statistics gathered from animations with motion blur. $\Delta$ indicates the step in key frames between the start and end position of the triangle. For each $\Delta$, we report the percentage of triangles potentially visible but culled by the STARTEND backface test, and $\bar{s}$ reports the maximum number of samples in any frame that comes from triangles falsely culled by the STARTEND test.

\subsection{Depth of Field}

For large apertures, the number of frontfacing triangles increases. The invalid test of only checking backface status in the center of the lens gives visible artifacts around silhouettes. This is shown in 

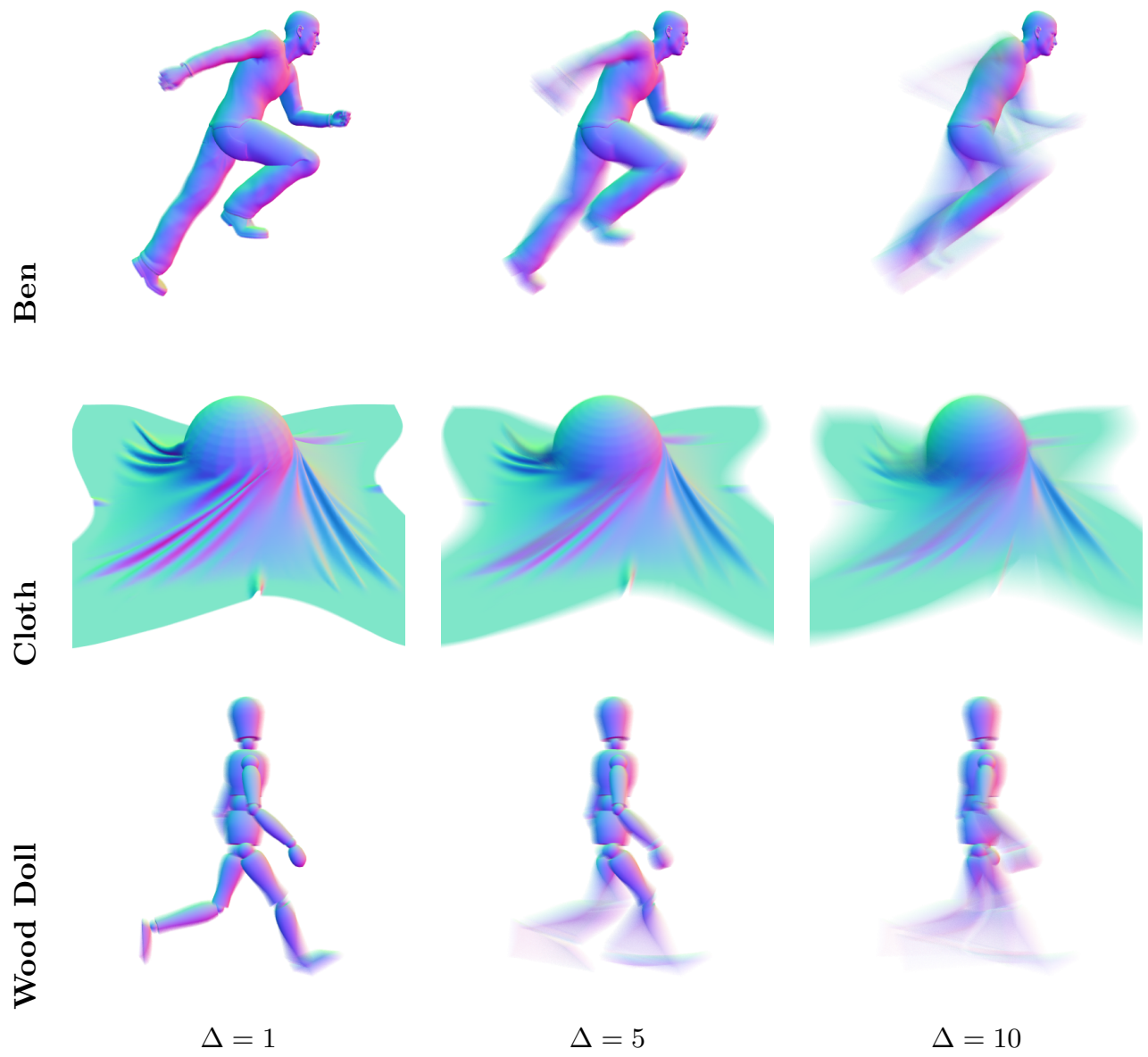

Figure 4: The test scenes with different animation speeds. $\Delta$ denotes the steps in keyframes in the animations.

Figure 6 . The correct test is inexpensive and we recommend that it is always enabled.

\subsection{Depth of Field and Motion Blur}

For the combined case of both motion blur and DOF, the vast majority of artifacts stems from DOF, so the tests should be tuned for a tight DOF test and a coarser motion blur test, as shown in Section 4.1. Figure 7 shows an example of this.

\section{References}

[Akenine-Möller et al. 07] Tomas Akenine-Möller, Jacob Munkberg, and Jon Hasselgren. "Stochastic Rasterization using Time-Continuous Triangles." In Graphics Hardware, pp. 7-16, 2007. 


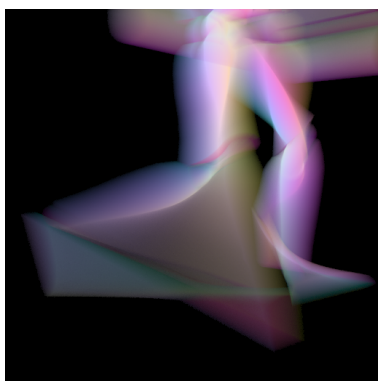

Correct BF

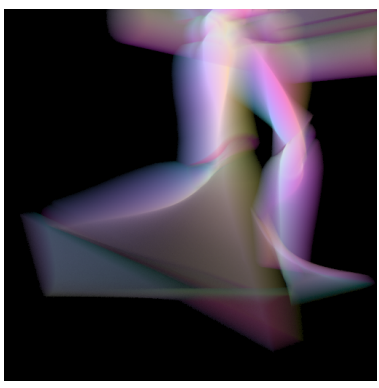

STARTEND

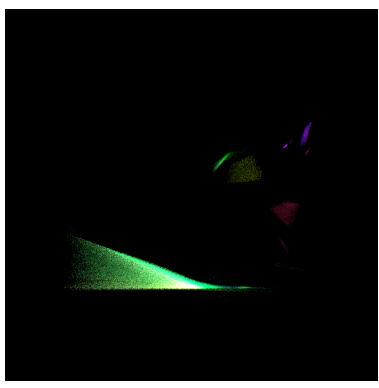

Diff image $(\times 20)$

Figure 5: A frame from the Wood Doll animation $(\Delta=10)$ with a camera rotating around the character ( $\pi / 2 \mathrm{rad} /$ frame), using $256 \mathrm{spp}$. In this example, the STARTEnd test falsely rejects about $6 \%$ of the triangles. In total, $11 \mathrm{k}(4 \%)$ pixels differ, $5.7 \mathrm{k}(2 \%)$ pixels fail a perceptual test and the PSNR is $47 \mathrm{~dB}$.

[Cook et al. 84] Robert L. Cook, Thomas Porter, and Loren Carpenter. "Distributed ray tracing." Computer Graphics (Proceedings of ACM SIGGRAPH 84) 18:3 (1984), 137-145.

[Gribel et al. 10] Carl Johan Gribel, Michael Doggett, and Tomas Akenine-Möller. "Analytical Motion Blur Rasterization with Compression." In High Performance Graphics, pp. 163-172, 2010.

[Makino and Berz 03] Kyoko Makino and Martin Berz. "Taylor Models and Other Validated Functional Inclusion Methods." International Journal of Pure and Applied Mathematics, 4:4 (2003), $379-456$.

[McCool et al. 02] Michael D. McCool, Chris Wales, and Kevin Moule. "Incremental and Hierarchical Hilbert Order Edge Equation Polygon Rasterization." In Graphics Hardware, pp. 65-72, 2002.

[Moore 66] R. E. Moore. Interval Analysis. Prentice-Hall, 1966.

[Olano and Greer 97] Marc Olano and Trey Greer. "Triangle Scan Conversion using 2D Homogeneous Coordinates." In Graphics Hardware, pp. 89-95, 1997.

[Ragan-Kelley et al. ] Jonathan Ragan-Kelley, Jaakko Lethinen, Jiawen Chen, Michael Doggett, and Fredo Durand. "Decoupled Sampling For Real-Time Graphics Pipelines." To appear in ACM Transactions on Graphics.

[Yee 04] Hector Yee. "A Perceptual Metric For Production Testing." Journal of Graphics Tools 9:4 (2004), 33-40. 

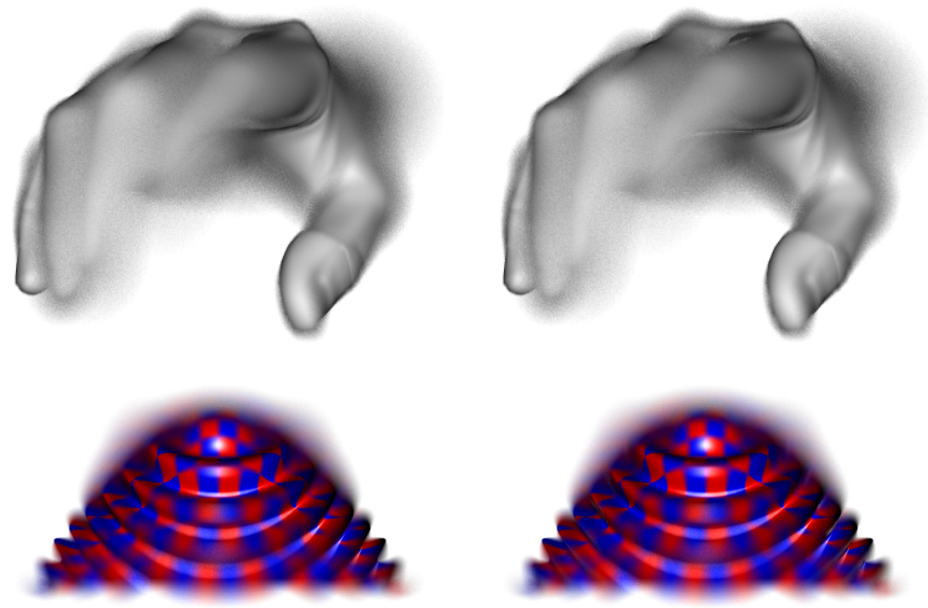

Exact backface test

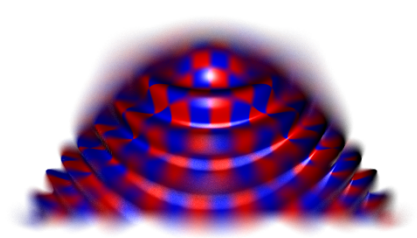

Center of lens BF test
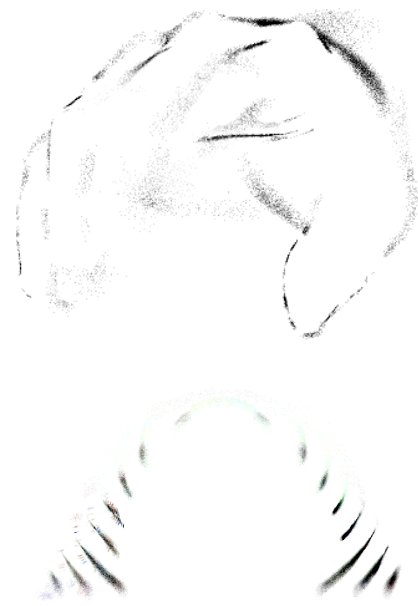

Difference $\times 10$

Figure 6: Two examples with large defocus blur with a correct backface test (left) and the center of the lens test (middle). Note the white streak in the blur on top of the hand in the middle image, and the missing occlusion on the right side of the displaced patch.

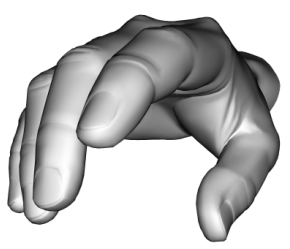

Sharp

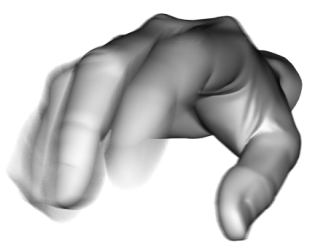

MB (0)

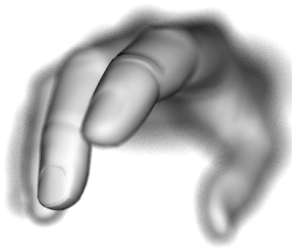

DOF (491)

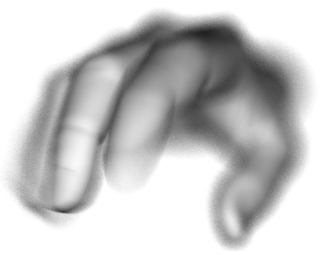

MB+DOF (604)

Figure 7: An example with both motion blur and depth of field. The numbers indicate how many triangles that are falsely culled by the incorrect backface test for each configuration. The model contains 15855 triangles. 